\title{
Statistical Channel Modeling of Overhead Low Voltage Broadband over Power Lines (OV LV BPL) Networks - Part 1: The Theory of Class Map Footprints of Real OV LV BPL Topologies, Branch Line Faults and Hook-Style Energy Thefts
}

\author{
Athanasios G. Lazaropoulos* \\ School of Electrical and Computer Engineering / National Technical University of Athens / \\ 9 Iroon Polytechniou Street / Zografou, GR 15780
}

Received January 22, 2020; Accepted February 26, 2020; Published March 12, 2020

\begin{abstract}
Due to the significant volatility of Broadband over Power Lines (BPL) networks regarding their circuital and topological characteristics, channel statistical modeling recently gains special attention from the BPL communications engineers. Among the recently presented channel attenuation statistical models, initial statistical hybrid model (iSHM) and modified statistical hybrid model (mSHM) have been theoretically defined and applied to overhead medium voltage (OV MV), underground medium voltage (UN MV) and overhead high voltage (OV HV) BPL networks so far. Apart from the iSHM and mSHM definition and application, the theory of the definition procedure of new virtual distribution and transmission BPL topologies, which describes the phases towards defining statistically equivalent BPL topologies and topology subclasses to the real indicative ones, has been demonstrated as well as the class maps, which are 2D capacity contour plots with respect to the channel attenuation statistical distributions (CASDs) parameters of iSHM and mSHM.

In this pair of papers, iSHM, mSHM, the definition procedure of new virtual BPL topologies and the class mapping are first applied to overhead low voltage (OV LV) BPL networks. Based on the class maps and the BPL topology database of Topology Identification Methodology (TIM), the required theory for illustrating the footprint of the real OV LV BPL topologies is first presented on class maps in this paper. On the basis of the class maps and the BPL topology database of Fault and Instability Identification Methodology (FIIM), the required theory for illustrating the footprint of the OV LV BPL topologies with branch line faults is first identified on class maps in this paper. On the basis of the class maps and the BPL topology database of hook style energy theft detection method (HS-DET method), the required theory for illustrating the footprint of the OV LV BPL topologies with a hook style energy theft is first demonstrated on class maps in this paper.
\end{abstract}

Keywords: Smart Grid; Broadband over Power Lines (BPL) networks; Power Line Communications (PLC); Distribution and Transmission Power Grids; Capacity; Statistics; Modeling 


\section{Nomenclature}

\begin{tabular}{|l|l|}
\hline AAAC & All Aluminum Alloy Conductor \\
\hline AWGN & Additive White Gaussian Noise \\
\hline BPL & Broadband over Power Lines \\
\hline BPMN & Business Process Model and Notation \\
\hline CASD & $\begin{array}{l}\text { Channel Attenuation Statistical } \\
\text { Distribution }\end{array}$ \\
\hline CDF & cumulative density function \\
\hline CS2 module & Coupling Scheme version 2 module \\
\hline DHM & deterministic hybrid model \\
\hline EMI & ElectroMagnetic Interference \\
\hline FIIM & Fault and Instability Identification \\
& Methodology \\
\hline FL noise model & Flat noise model \\
\hline HS-DET method & hook style energy theft detection method \\
\hline HV & High Voltage \\
\hline ICT & Information and Communication \\
& Technology \\
\hline IPSD limits & injected power spectral density limits \\
\hline IP & Internet Protocol \\
\hline iSHM & initial Statistical Hybrid Model \\
\hline LOS & Line-of-Sight \\
\hline LV & Low Voltage \\
\hline L1PMA & L1 Piecewise Monotonic Approximation \\
\hline MLE & Maximum Likelihood Estimator \\
\hline mSHM & modified Statistical Hybrid Model \\
\hline MTL & multiconductor transmission line \\
\hline MtM & MultiWire-to-MultiWire \\
\hline MV & Medium Voltage \\
\hline OV & Overhead \\
\hline PES & Percent Error Sum \\
\hline PSD & Power spectral density \\
\hline SG & Smart Grid \\
\hline SHM & Statistical Hybrid Model \\
\hline TIM & Topology Identification Methodology \\
\hline TL & Transmission Line \\
\hline TM2 method & Transmission Matrix version 2 method \\
\hline UN & Underground \\
\hline WtG & Wire-to-Ground \\
\hline WtW & Wire-to-Wire \\
\hline & \\
\hline
\end{tabular}




\section{Introduction}

During the recent years, the traditional power grid, which represents an omnipresent widely branched hierarchical network structure and has been designed to facilitate the one-way power delivery from producers to consumers with relatively few one-way communications modalities is transformed into the smart grid, which is based on the traditional power grid infrastructure and may support two types of flows; say, a two-way power and a two-way information flow [1]-[3]. As the two-way information flow is concerned in this paper, the smart grid can be treated as an advanced IP-based communications network where a plethora of broadband applications can be supported that can facilitate either power utilities (e.g., real-time monitor, meter and control of the power grid equipment and wired infrastructure) or customers (e.g., real-time monitor and control of their power flows). To implement the two-way information flow across the smart grid, BPL technology attracts great attention from the stakeholders among the available communications technology proposals since it exploits the already installed wired power grid infrastructure [4]-[6]. However, transmission and distribution power grids have intentionally been designed to deliver power and for that reason power grids are a hostile medium for communications signals, such as BPL ones, through their infrastructure and equipment [7]-[11].

High and frequency-selective channel attenuation is considered as one of the critical inherent deficiencies of the BPL signal propagation and transmission across the power grid, thus requiring an accurate BPL channel model to be developed. So far, two main categories of BPL channel models are available: the deterministic and statistical BPL channel models. Here, it should be noted that statistical BPL channel models are based on the deterministic BPL channel models to a large degree. Hence, as the deterministic BPL channel attenuation modeling is first concerned, the relevant deterministic BPL channel models typically follow either a bottom-up approach or a top-down approach or hybrid approaches that synthesize the aforementioned bottom-up and top-down ones [7], [8], [12]-[23]. Among the available deterministic BPL channel models of the literature, DHM is selected for the analysis of this paper due to its exhaustive validation in various MTL configurations of transmission and distribution BPL networks [7], [8], [12], [24]-[30]. DHM consists of two interconnected modules, say: (i) the bottom-up approach module that is based on the formality of the MTL theory and its interaction with various similarity transformations; and (ii) the top-down approach module (TM2 method) that is based on the handling of the multidimensional transmission matrices of the cascaded BPL topologies of the examined BPL networks and topologies, the coupling scheme module, which determines the way that the BPL signal is injected/extracted onto/from power lines, and the capacity module that computes the highest information rate without errors that can be achieved in the examined BPL networks. Among the broadband performance metrics that are available as output from the DHM, channel attenuation and capacity are of interest during the application of the statistical BPL channel model of this pair of papers. Apart from the aforementioned deterministic framework, that is anyway required for the analysis of this pair of papers, the attention of BPL communications engineers and scientists regarding the BPL channel attenuation modeling has also been focused on statistical BPL channel models. So far, a great number of statistical BPL channel models, which are based on the results 
of deterministic BPL channel models, has been proposed in a variety of BPL technology application fields [31]-[42]. SHM, which has been proposed in [35]-[37], is based on DHM and is hereafter applied. In fact, iSHM and mSHM, which are the two versions of the SHM, are both based on DHM while their simulation results may be considered as the statistically processed DHM numerical results through a set of appropriate CASDs; say, iSHM applies Gaussian, Lognormal, Wald, Weibull and Gumbel CASDs while mSHM applies Empirical CASD. By combining the channel attenuation results of DHM and CASDs through a six-phase flowchart procedure, iSHM and mSHM may give as output capacity ranges for given MTL configuration, IPSD limit, noise PSD level, coupling scheme and BPL topology subclass where a BPL topology subclass is uniquely characterized by its respective real indicative BPL topology and consists of statistically equivalent BPL topologies to the indicative one in terms of the MLE parameters of the applied CASDs (CASD MLEs). The capacity simulation results of iSHM and $\mathrm{mSHM}$ are based on real indicative BPL topologies whose topological results are well defined in terms of the topology length, the interconnections between branches / main lines, branch lengths, branch distances and branch terminations. Depending on the average value of their capacity range, BPL topology subclasses can be further classified into five BPL topology classes; say, LOS, rural, suburban, urban A and urban B BPL topology classes regardless of the type of power grid. In accordance with [36], [37], [45], it should be here noted that the capacity estimation success and the total simulation time of CASDs for given SHM version have been benchmarked for different transmission and distribution BPL networks revealing the strong dependence of the capacity estimation success on the applied CASD and SHM version. In fact, the definition procedure of virtual indicative BPL topologies, which has been analyzed in [43]-[45] and is hereafter simply denoted as definition procedure, enriches BPL topology classes with BPL topology subclasses whose respective virtual indicative BPL topologies are not defined in terms of the topology length, the interconnections between branches and main lines, branch lengths, branch distances and branch terminations but are only statistically defined in terms of the applied SHM version and its corresponding successful CASD MLEs. The definition procedure is a three-group eleven-step flowchart procedure that defines the MLE parameter ranges for given CASD and SHM version while its output is the class map, which is a $2 \mathrm{D}$ contour plot, that illustrates the borders between the BPL topology classes and corresponds each CASD MLE parameter pair to its BPL topology subclass average capacity for given power grid type, SHM version, CASD, coupling scheme, IPSD limits and noise levels. The simulation results of the definition procedure are presented for distribution and transmission BPL networks in [44] and [45], respectively, when the most successful CASDs for given SHM version are adopted.

In this set of papers, iSHM, mSHM, the definition procedure and the class maps are first applied to OV LV BPL networks. By exploiting the already acquired application experience of SHM to OV MV, UN MV and OV HV BPL networks [36], [37], [45], new results are provided for OV LV BPL topologies thus: (i) revealing the most successful CASD among the aforementioned ones for iSHM and mSHM; and (ii) benchmarking the performance of iSHM and mSHM.

But the main contribution of this set of papers is the proposal of the footprint areas on the class maps, hereafter simply denoted as footprints, that allows the impact investigation of critical events that may occur during the operation of OV LV power 
grids. To determine a footprint in the class maps, the OV LV BPL topology databases of three smart grid broadband applications that are supported by the BPL technology (e.g., TIM [46], FIIM [46] and HS-DET method [47]) are here retrieved and applied. First, since the class maps are 2D contour plots that are curved with respect to CASD MLEs for given CASD, the average capacity results of all the possible BPL topology subclasses are grouped among the five BPL topology classes and are demonstrated on class maps into five BPL topology class areas. By exploiting the BPL topology database of TIM that contains all the possible real OV LV BPL topologies, respective OV LV BPL subclasses are defined and their average capacities are computed with respect to the most successful CASDs of iSHM and mSHM. Then, the footprint of the real OV LV BPL topologies is going to be first illustrated on the class maps thus separating the real OV LV BPL topology footprint from the virtual one. Second, a critical issue concerning the supply security and the fine operation of the OV LV power grid is the immediate response and repair to faults that may occur across the OV LV power grid infrastructure. In this paper, by exploiting the BPL topology database of FIIM, the impact of a single branch line fault across an OV LV BPL topology is first assessed through the footprint of the OV LV BPL topologies with branch line faults on class maps for given indicative OV LV BPL topology. Third, energy theft defines a multi-billion problem for power utility companies either in traditional power systems or in the emerging smart grid. In this paper, by exploiting the BPL topology database of HS-DET method, the impact of the hook style energy theft across an OV LV BPL topology is first assessed through the footprint of the OV LV BPL topologies with a single hook for energy theft on class maps for given indicative OV LV BPL topology. In this paper, the theory that is required for the introduction of the footprint concept for real OV LV BPL topologies, OV LV BPL topologies with branch line faults and OV LV BPL topologies with hook-style energy thefts is detailed.

The rest of this paper is organized as follows: In Section II, the OV LV MTL configuration is presented as well as the real indicative OV LV BPL topologies of this pair of papers. Section III summarizes the basics of DHM, iSHM, mSHM, the definition procedure and the class maps as well as the required settings for their default operation during the study of OV LV BPL topologies. Section IV briefly presents TIM, FIIM and HS-DET method as well as the required settings for the update of the respective databases. Also, the theory concerning the iSHM and mSHM class maps footprints of real OV LV BPL topologies, of OV LV BPL topologies with branch line faults and of OV LV BPL topologies with hook-style energy thefts is carefully presented. Section V concludes this paper.

\section{OV LV MTL Configuration and Indicative OV LV BPL Topologies}

Due to the physical properties of OV LV TLs and the involved power grid equipment, the BPL signal propagation and transmission across the OV LV power grid considerably differs from the one of well-known communications media such as twistedpair, coaxial or fiber-optic cables. This Section presents the applied OV LV MTL configuration and indicative OV LV BPL topologies that mainly affect the BPL signal propagation and transmission, respectively. 


\subsection{OV LV MTL Configuration}

In Fig. 1(a), the typical OV LV MTL configuration that is examined in this paper is illustrated. The examined OV LV MTL configuration consists of four parallel noninsulated conductors (i.e., $n^{\text {ovLV }}=4$ ), which are spaced each other by a vertical distance $\triangle \mathrm{ovLV}$ in the range from $0.3 \mathrm{~m}$ to $0.5 \mathrm{~m}$. The upper conductor of radius $r \mathrm{OVLV}, \mathrm{n}$ is the neutral one, while the lower three conductors of radius rovLv,p stand for the three phases.

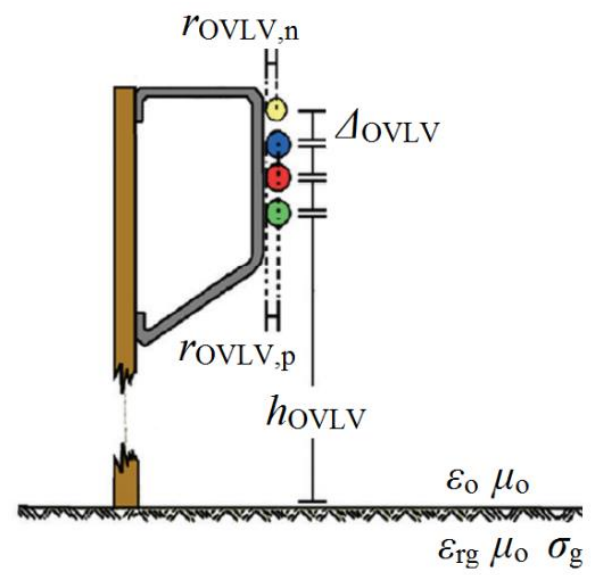

(a)

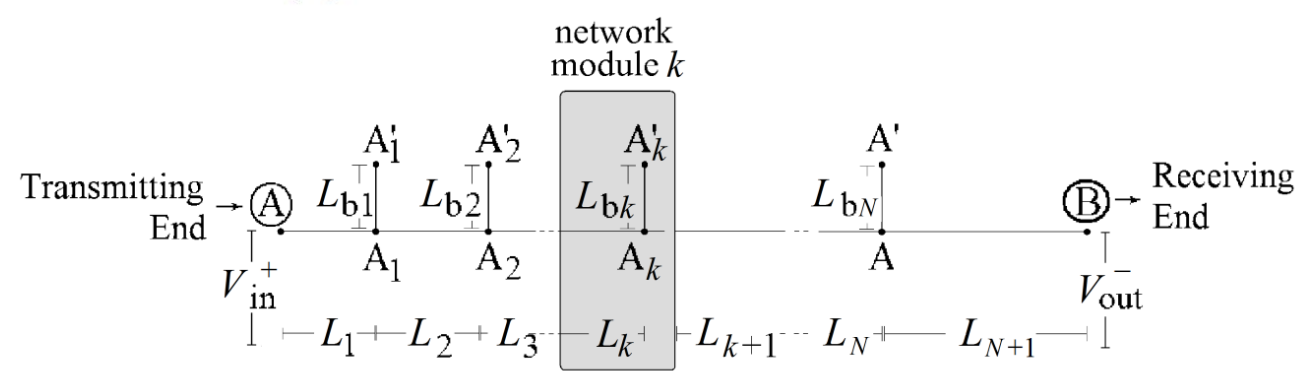

(b)

Fig. 1. (a) OV LV MTL configuration [12]. Typical OV LV BPL topology with $N$ branches [35].

Note that the lowest phase conductor is hung at height hovLv in the range from 6 $\mathrm{m}$ to $10 \mathrm{~m}$ above the ground. As the dimension, the material and the structure of conductors are concerned, the three-phase four-conductor OV LV MTL configuration consists of $3 \times 54.6 \mathrm{~mm}^{2}+1 \times 34.4 \mathrm{~mm}^{2}$ AAACs [12], [48]. The ground is considered as the reference conductor with conductivity $\sigma_{\mathrm{g}}$ and relative permittivity $\varepsilon_{\mathrm{rg}}$ that are equal to 5 $\mathrm{mS} / \mathrm{m}$ and 13, respectively [7], [12], [15], [23], while the impact of imperfect lossy ground on BPL signal propagation over OV power lines has been analyzed in [49]-[51].

\subsection{Real Indicative OV LV BPL Topologies}

To study OV LV BPL networks, each network is assumed to be divided into cascaded OV LV BPL topologies. A typical OV LV BPL topology is illustrated in Fig. 1(b). Each OV LV BPL topology is bounded by the transmitting and receiving ends 
where BPL devices, such as BPL signal injector, BPL signal extractor and BPL signal repeater, are installed depending on the relative location of the topology across the network. Across the BPL signal transmission path, $N$ branches with their respective terminations may be encountered. The arbitrary $k$ branch has length equal to $L_{b} k$, $k=1, \ldots, N$ and is located at distance $\sum_{i=1}^{k} L_{\mathrm{b} k}$ from the transmitting end. In accordance with TM2 method of DHM [12], [24], each OV LV BPL topology is treated as a concatenation of $N+1$ network modules; say, each of the first $N$ network modules comprises the $k$ branch and the TL of length $L_{k}$, which is the distance between the examined branch and its previous one, while the last network module only comprises the TL of length $L_{N+1}$, which is the distance between the last branch and the receiving end.

In accordance with [12], [47], [52]-[54], five OV LV BPL topology classes (i.e., LOS, rural, suburban, urban A and urban B) with their respective representative BPL topologies can be defined so that a thorough study of all OV LV BPL topologies may be fulfilled during DHM application. In Table 1, the representative OV LV BPL topologies of the respective five OV LV BPL topology classes (real indicative OV LV BPL topologies) are reported in the case of DHM as well as their topological characteristics. Similarly, when SHM is applied the representative OV LV BPL topologies of Table 1 act as the representative ones for the respective main subclasses of the OV LV BPL topology classes. The selection of the main subclass for given OV LV BPL topology class remains critical during the preparation of class maps since its capacity influences the capacity boundaries among the topology classes.

\section{The Basics of DHM, SHM, Definition Procedure and Class Maps}

Class maps, which are the main graphical metric in order to access the case studies of this pair of papers, are the output of the definition procedures of iSHM and mSHM, which are anyway the two versions of SHM. Apart from the required statistical processing, SHM is based on DHM results that are the channel attenuation and the capacity. In this Section, a brief synopsis of DHM, SHM, definition procedure and class maps is provided as well as their interoperability.

\subsection{DHM}

In this paper, DHM can be treated as the module concatenation of the bottom-up, the top-down, the coupling scheme and the capacity computation modules.

On the basis of its first two interconnected modules, say, the bottom-up and the top-down approach module, several useful intermediate metrics, among them is the line channel transfer function matrix, may be calculated by DHM for given OV LV MTL configuration and OV LV BPL topology. Since $n^{\text {OVLV }}$ phase conductors that are greater than 2 are encountered in OV LV MTL configurations, the standard TL analysis is extended by DHM to the MTL analysis. Extensively been analyzed in [7], [8], [12], [25], [26], [27], [52], [55]-[58], bottom-up module of DHM computes the $n^{\text {ovLV }}$ modes that are supported and propagate across the OV LV MTL configurations in the frequency range of interest. By receiving the results of the bottom-up module and applying TM2 method of the top-down module, the $n^{\text {ovL }} \times n^{\text {ovL }}$ line channel transfer function matrix $\mathrm{H}^{\{} \cdot \mathrm{f}$ that relates line quantities with modal ones is given by 


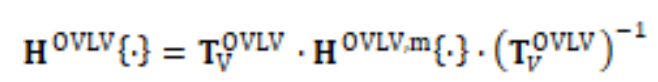

where $\mathbf{H}^{\mathrm{OVLV}, \mathrm{m}}\{\mathrm{x}\}$ is the $n^{\mathrm{OVLV}} \times n^{\mathrm{OVLV}}$ modal channel transfer function matrix that mainly depends on the examined OV LV MTL configuration and OV LV BPL topology and $\mathbf{T}_{\mathrm{V}}^{\text {OVLV }}$ is a $n^{\text {OVLV }} \times n^{\text {OVLV }}$ matrix that depends on the frequency, the physical properties of the TLs and the geometry of the OV LV MTL configuration.

As the line channel transfer function matrix is computed by the top-down module of DHM, the coupling scheme module can implement different coupling schemes that define the practical way that the signals are injected into and extracted from the TLs of the OV LV BPL networks. With reference to [29], [59], CS2 module, which is the most

Table 1

Representative OV LV BPL Topologies (Real indicative OV LV BPL Topologies) for DHM and SHM

\begin{tabular}{|c|c|c|c|c|}
\hline $\begin{array}{c}\text { OV LV BPL } \\
\text { Topology Class }\end{array}$ & $\begin{array}{c}\text { OV LV BPL } \\
\text { Topology Name } \\
\text { (and OV LV BPL } \\
\text { Topology Subclass } \\
\text { Name) }\end{array}$ & $\begin{array}{l}\text { Number } \\
\text { of } \\
\text { Branches } \\
(N)\end{array}$ & Length of Main Lines & Length of Branches \\
\hline $\begin{array}{l}\text { Typical urban } \\
\text { topology class }\end{array}$ & $\begin{array}{l}\text { Urban case A } \\
\text { (main subclass) }\end{array}$ & 3 & $\begin{aligned} L_{1}=500 \mathrm{~m}, L_{2} & =200 \mathrm{~m}, L_{3}=100 \mathrm{~m}, \\
L_{4} & =200 \mathrm{~m}\end{aligned}$ & $L_{\mathrm{b} 1}=8 \mathrm{~m}, L_{\mathrm{b} 2}=13 \mathrm{~m}, L_{\mathrm{b} 3}=10 \mathrm{~m}$ \\
\hline $\begin{array}{c}\text { Suburban } \\
\text { topology class }\end{array}$ & $\begin{array}{l}\text { Suburban case } \\
\text { (main subclass) }\end{array}$ & 2 & $L_{1}=500 \mathrm{~m}, L_{2}=400 \mathrm{~m}, \mathrm{~L}_{3}=100 \mathrm{~m}$ & $L_{\mathrm{b} 1}=50 \mathrm{~m}, L_{\mathrm{b} 2}=10 \mathrm{~m}$ \\
\hline $\begin{array}{l}\text { Rural topology } \\
\text { class }\end{array}$ & $\begin{array}{c}\text { Rural case } \\
\text { (main subclass) }\end{array}$ & 1 & $L_{1}=600 \mathrm{~m}, L_{2}=400 \mathrm{~m}$ & $L_{\mathrm{b} 1}=300 \mathrm{~m}$ \\
\hline $\begin{array}{l}\text { LOS topology } \\
\text { class }\end{array}$ & $\begin{array}{c}\text { LOS case } \\
\text { (main subclass) }\end{array}$ & 0 & $L_{1}=1000 \mathrm{~m}$ & - \\
\hline
\end{tabular}

recently upgraded coupling scheme module for BPL networks, is adopted in this paper. CS2 module can describe the BPL signal coupling procedure using two interfaces, namely: (i) BPL signal injection; and (ii) BPL signal extraction interface while it can support three types of coupling schemes for the OV LV BPL networks, namely: (1) Coupling Scheme Type 1: WtG coupling scheme; (2) Coupling Scheme Type 2: $W t W$ coupling scheme; and (3) Coupling Scheme Type 3: MtM coupling scheme. With reference to eq. (1), CS2 module computes the coupling scheme channel transfer function that relates output and input BPL signal through

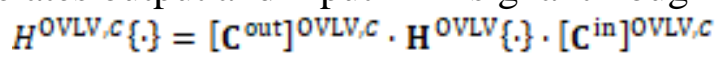

for given coupling scheme where $[\cdot]^{c}$ denotes the applied coupling scheme, $\boldsymbol{c}^{\text {in }}$ is the input coupling $n^{\text {ovLv }} \times 1$ column vector that deals with the BPL signal injection interface and $\mathbf{c}^{\text {out }}$ is the ouput coupling $1 \times n^{\text {ovLy }}$ line vector that deals with the BPL signal extraction interface. Different coupling schemes involve different conductors of the examined OV LV MTL configuration and entail corresponding power restrictions and thus corresponding $\mathbf{C}^{\text {in }}$ and $\mathbf{C}^{\text {out }}$ as explained in [59], [29].

As the coupling scheme channel transfer function is well defined by eq.(2) for given coupling scheme, the last module of DHM is the capacity one that computes the maximum achievable transmission rate that can be reliably transmitted for given OV LV BPL topology and remains the crucial measure for the class maps. Apart from the 
coupling scheme channel transfer function of eq. (2), two other critical parameters (i.e., IPSD limits and noise PSD levels) should be defined so that the capacity of a given OV LV BPL topology can be determined, namely:

- IPSD limits: Since BPL systems operate in a common frequency range with other already licensed communications services, by adopting suitable IPSD limits the unintentional EMI to the other already licensed communications services (e.g., aeronautical radionavigation, radio astronomy, mobile satellite and maritime mobile) can be significantly mitigated. Among the most noted proposals that define suitable EMI policies and thus respective IPSD limits are FCC Part 15, German Reg TP NB30 and the BBC / NATO Proposal. More analytically, the electric field strength limits proposed by the aforementioned proposals are presented in [10], [60], [61] while the respective IPSD limits are determined in [61] for OV BPL networks.

- Noise PSD levels: OV BPL networks suffer from colored background and impulsive noise [7], [12], [25], [27], [52], [53], [55], [56]. However, FL noise model of [62] suggests that the assumption of AWGN PSD levels can remain a simple but accurate noise approximation during the capacity computations of $\mathrm{OV}$ LV BPL networks in the $3-30 \mathrm{MHz}$ frequency that is anyway the frequency range of interest of this paper. Hence, the AWGN PSD level of OV LV BPL networks that is adopted in this paper is assumed to be equal to $-60 \mathrm{dBm} / \mathrm{Hz}$.

Since the coupling scheme channel transfer function, IPSD limits and noise PSD levels are well defined for given MTL configuration, OV LV BPL topology, applied coupling scheme, EMI policy and the noise environment, the capacity $C$ is given by [52], [55], [56]

$$
\begin{aligned}
& C=f_{s} \Sigma_{q=1}^{Q} \log _{2}\left\{1+\left[\frac{\left(p\left(f_{q}\right)_{L}\right.}{\operatorname{lv}\left(f_{q}\right)_{L}} \cdot\left|H^{\mathrm{OVLW}} \mathrm{C}_{2}\left(f_{q}\right)\right|^{2}\right]\right\} \\
& f_{q}=3 \mathrm{MHz}+(q-1) \cdot f_{s}, q=1, \ldots, Q \\
& \mathbf{f}=\left[\begin{array}{lllll}
f_{1} & \cdots & f_{q} & \cdots & f_{Q}
\end{array}\right], q=1_{s, \ldots}, Q
\end{aligned}
$$

where $f_{q}$ is the flat-fading subchannel start frequency, $f_{s}$ is the flat-fading subchannel frequency spacing, $Q$ is the number of subchannels in the examined 3-30 MHz frequency range, $\mathbf{f}$ is the $1 \times Q$ line vector that consists of the flat-fading subchannel start frequencies $f_{q}, p(*)$ is the applied IPSD limits in $\mathrm{dBm} / \mathrm{Hz}, N(*)$ is the applied AWGN PSD levels in $\mathrm{dBm} / \mathrm{Hz}$ and \langle\rangle$_{\mathrm{L}}$ is an operator that converts $\mathrm{dBm} / \mathrm{Hz}$ into a linear power ratio $(\mathrm{W} / \mathrm{Hz})$.

\subsection{SHM}

SHM, which consists of iSHM and mSHM that are its two versions, has been proposed in [35]-[37]. SHM exploits the deterministic results of DHM while SHM can be considered as a statistical BPL channel model with its recent application to various transmission and distribution BPL networks of interest [35]-[37]. The BPMN diagrams of iSHM and mSHM that describe their operation and the transformation of the DHM deterministic results to the SHM simulation results of this paper are demonstrated in Figs. 2(a) and 2(b), respectively. Either iSHM or mSHM consists of six Phases (Phase A-F) while each Phase is graphically constrained by a grey container and is characterized by its corresponding procedure and produced files shown in light blue color. The red elements of the BPMN diagrams are analyzed in Sec.3.3 since these are modifications required by the definition procedure. Significant similarities and 
differences between iSHM and mSHM occur and they are analytically highlighted in this subsection.

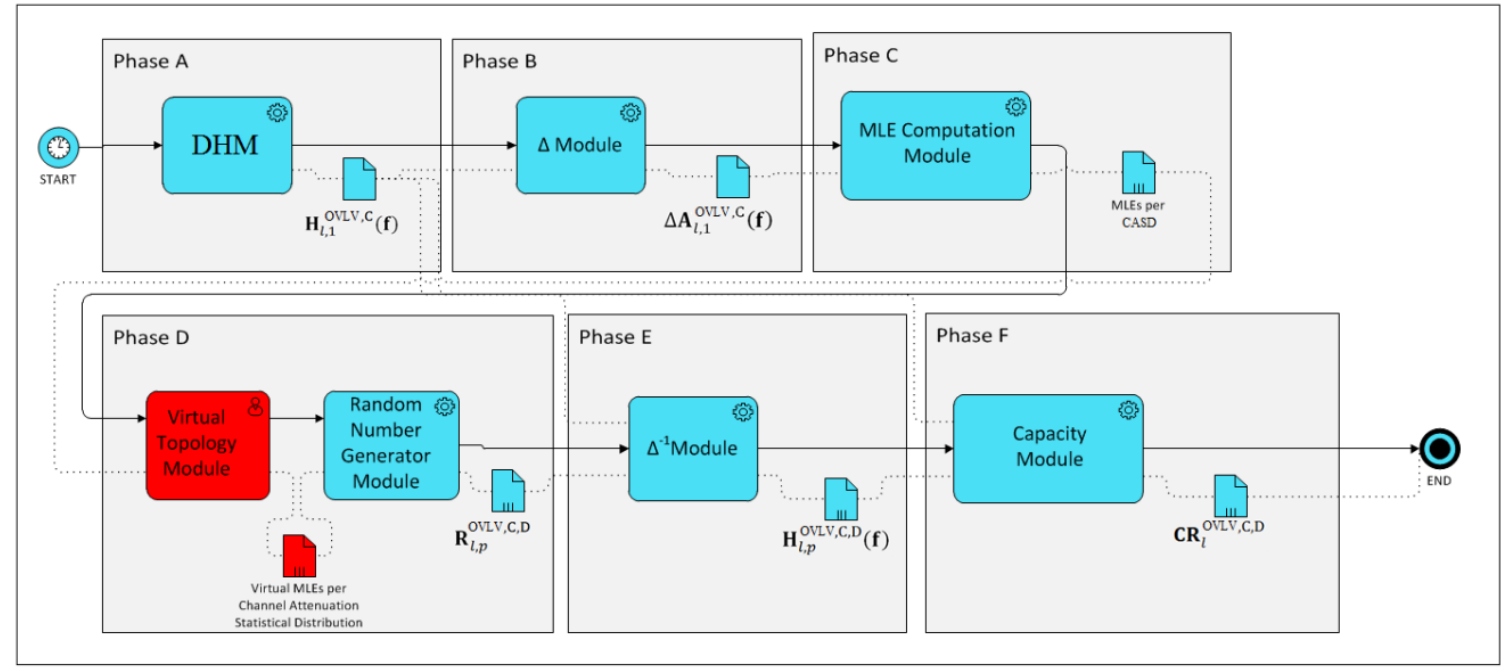

(a)

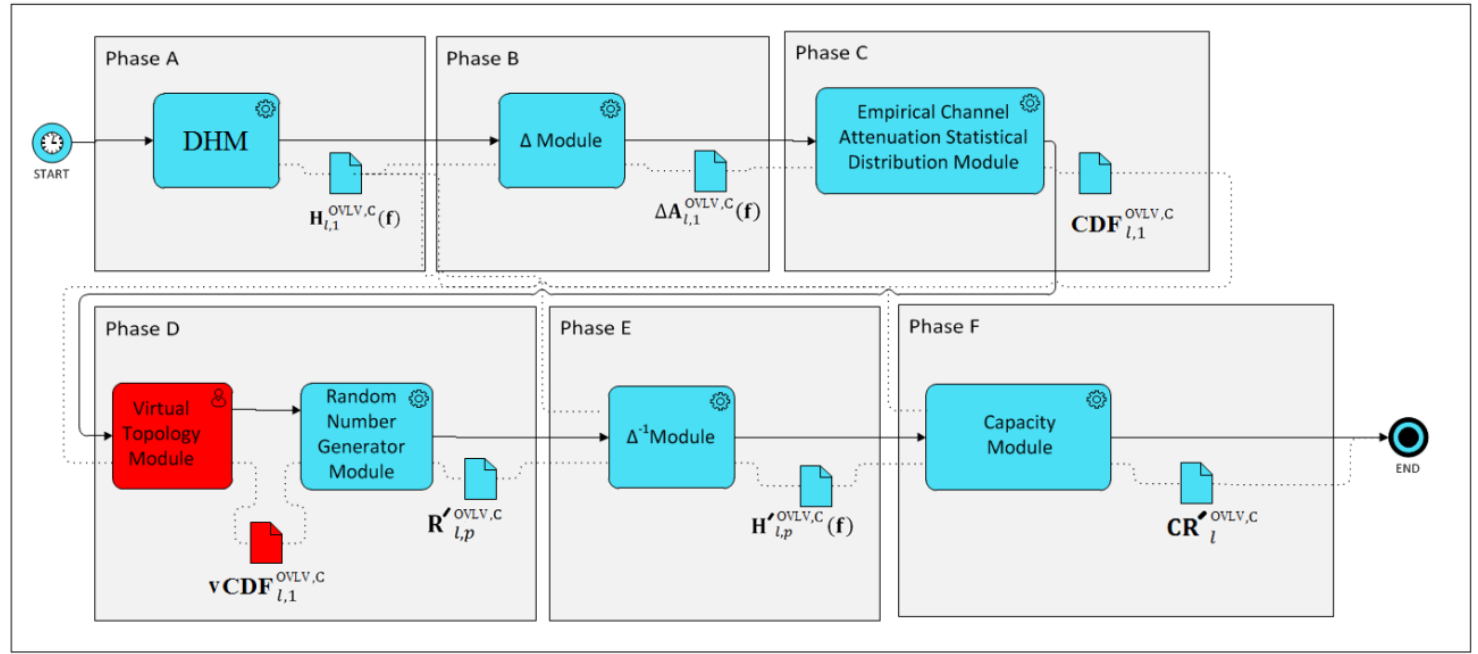

(b)

Fig. 2. BPMN diagrams of SHM. (a) iSHM [35]. (b) mSHM [37].

In accordance with [35] and with reference to Fig. 2(a), the operation of iSHM can be described through the concatenation of the Phases A-F. More specifically, Phase A of iSHM deals with the bottom-up, the top-down and the coupling scheme modules of DHM by taking as input the $l$ real indicative OV LV BPL topology of Table 1, its corresponding OV LV MTL configuration and the applied coupling scheme while it gives as output the $1 \times Q$ coupling scheme channel transfer function line vector

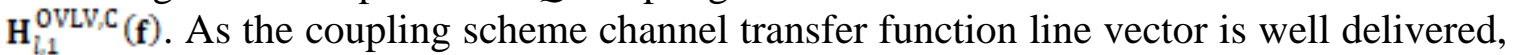
Phase $\mathrm{B}$ of iSHM computes the $1 \times Q$ channel attenuation difference line vector $\Delta \mathrm{A}_{\mathbb{A}_{1}}^{\mathrm{OVVW}} \mathrm{C}_{(\mathbf{f})}$ of the $l$ real indicative OV LV BPL topology with respect to the LOS case for given 
coupling scheme through its $\Delta$ module. Then, Phase $\mathrm{C}$ of iSHM computes the MLEs of the supported iSHM CASDs (i.e., Gaussian, Lognormal, Wald, Weibull and Gumbel CASDs) by applying MLE computation module for given channel attenuation difference line vector. Note that the MLE estimation method of the five supported iSHM CASDs is presented in Appendix A of [35] as well as the respective CDFs. By deploying the random number generator module, Phase $\mathrm{D}$ of iSHM gives as output the $1 \times Q$ random number line vector $\mathbf{R}_{l, p}^{\mathrm{OVLV}, \mathrm{C}_{\mathrm{D}} \mathrm{D}}$ for given indicative OV LV BPL topology and coupling scheme where $\left[{ }^{*}\right]^{\mathbb{D}}$ denotes the applied CASD and $p, p=1, \ldots, P+1$ is the member number in the OV LV BPL topology main subclass. Thus, each OV LV BPL main subclass finally comprises its real indicative OV LV BPL topology $(p=1)$, which is one of the first four OV LV BPL topologies of Table 1 , and $P$ statistically equivalent virtual OV LV BPL topologies in terms of the CASD MLEs of the real indicative OV LV BPL topology. Phase E of iSHM performs the inverse procedure of Phase B through its $\Delta^{-1}$ module by computing $1 \times Q$ coupling scheme channel transfer function line vector $\mathrm{H}_{l, p}^{\mathrm{OVW} \mathrm{C}_{\mathrm{D}} \mathrm{D}}(\mathrm{f})$ of each of the $P$ members of each BPL topology main subclass for the examined coupling scheme and CASD. Finally, Phase F of iSHM computes

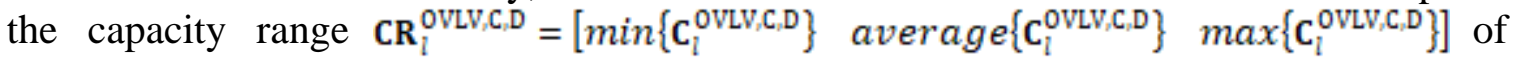
each OV LV BPL topology main subclass for given coupling scheme and CASD by applying capacity module of DHM where

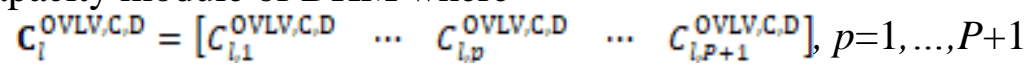

is the capacity line vector of the examined OV LV BPL topology main subclass, $C_{l p p}^{\text {OVLV } \mathrm{C} D}$ is the capacity of the $p$ member of the examined OV LV BPL topology main subclass, $\min \{x\}$, average $\{x\}$ and $\max \{x\}$ computes the minimum, the average and the maximum value of an array, respectively. More details concerning the operation of iSHM are given in [35].

In accordance with [37] and with reference to Fig. 2(b), the operation of mSHM can be described by revealing the similarities and the differences between mSHM Phases and the respective ones of iSHM. Phases A and B of mSHM remain the same with the respective Phases of iSHM. The main differences between $\mathrm{mSHM}$ and iSHM are concentrated in Phases C and D; since mSHM adopts the Empirical CASD instead of the five CASDs of iSHM, the Empirical channel attenuation statistical distribution module is deployed in Phase $\mathrm{C}$ for $\mathrm{mSHM}$ that receives as input the coupling scheme channel attenuation difference and it gives as output the Empirical CDF of the coupling scheme channel attenuation difference $\mathbf{C D F}_{l_{i} 1}^{\mathrm{OVLV}_{i} \mathrm{C}}$ for given OV LV BPL topology and coupling scheme. Then, the random number generator of mSHM Phase D gives as output the random number $1 \times Q$ line vector $\mathbf{R}_{h_{b} p}^{\mathrm{OVLV}_{2} \mathrm{C}}$ for given coupling scheme and indicative distribution BPL topology after the inverse interpolation to achieve the Empirical CDF projection of the random values taken by Phase C. Similarly to iSHM, mSHM Phase E computes the $1 \times Q$ coupling scheme channel transfer function line vector $\mathrm{H}_{\mathrm{l}, \mathrm{p}}^{\mathrm{OOVW}}, \mathrm{C}_{(\mathrm{f})}$ of each of the $P$ members of each BPL topology main subclass for the examined coupling scheme while mSHM Phase F computes the capacity range $\mathrm{CR}_{0}^{\text {rovLV }}$ of each OV LV BPL topology main subclass for given coupling scheme in accordance with the iSHM capacity range $\mathbf{C R}_{\mathbb{l}}^{\mathrm{OVLW}} \mathrm{C}_{\mathrm{D}}$ and eq. (6) when Empirical CASD is applied. 


\subsection{The Definition Procedure and Class Maps}

In accordance with Sec. 3.2., when the topological and circuital characteristics of real OV LV BPL topologies are available, iSHM and $\mathrm{mSHM}$ may define the respective BPL topology subclasses and compute the corresponding capacity ranges. As already been identified in [43]-[45], the underrepresentation of the BPL topology classes during the BPL statistical channel modelling can be mitigated through the insertion of virtual indicative BPL topologies and their respective subclasses through the adoption of the definition procedure. In [43], the theoretical framework of the definition procedure has been detailed while in Figs. 3(a) and 3(b) the flowcharts of the definition procedure of iSHM and $\mathrm{mSHM}$ are demonstrated, respectively. Note that the definition procedure imposes the operation of virtual topology module with its corresponding file output (i.e., red elements of BPMN diagrams) in Figs. 2(a) and 2(b) for iSHM and mSHM, respectively.

In accordance with [43] and with reference to Fig. 3(a), iSHM definition procedure flowchart consists of eleven steps; nine of them (i.e., FL1.02-FL1.10) are categorized into three groups (i.e., Group 1.A-1.C) while the first and eleventh steps (i.e., FL1.01 and FL1.11) deal with the definition of main subclasses and the graphical preparation of class maps, respectively. Since the real indicative OV LV BPL topologies of main subclasses of Table 1 have already been delivered by the step FL1.01, Group 1.A computes the capacities of these topologies $C_{l}^{\mathrm{OVLV}, \mathrm{C}}, l=1,2,3,4,5$ and the capacity borders between the adjacent OV LV BPL topology classes $C B o_{l}^{\text {OVLV }, \mathrm{C}}, l=1,2,3,4$ in accordance with eq.(1) of [43]. At the same time, Group 1.B, which consists of five steps (i.e., FL1.04-FL1.08), first computes each MLE pair $\left(M L E_{-} 1_{l}^{\mathrm{OVLV}_{l} \mathrm{C}_{2} \mathrm{D}}\right.$ and $\left.M L E_{-} 2_{l}^{\mathrm{OVLV}_{l} \mathrm{C}_{2} \mathrm{D}}, l=1,2,3,4,5\right)$ of the real indicative OV LV BPL topologies of the main subclasses per CASD at step FL1.04. Second, the step FL1.05 of Group 1.B defines the presentation resolution of the upcoming class maps by computing the length of horizontal spacings $\triangle M L E_{-} 1_{l}^{\text {OVLV, } \mathrm{C}_{3} \mathrm{D}}, l=1,2,3,4,5$ and of vertical spacings $\triangle M L E \_2_{l}^{\text {OVLV }_{a} \mathrm{C} D}, l=1,2,3,4,5$ by taking into account the number of spacings for the horizontal axis no_1 and vertical axis no_2, respectively. Third, step FL1.06 computes all (no_1 1 1) $\times($ no_2 +1$)$ MLE pair combinations of the virtual indicative OV LV BPL topologies of virtual subclasses per CASD

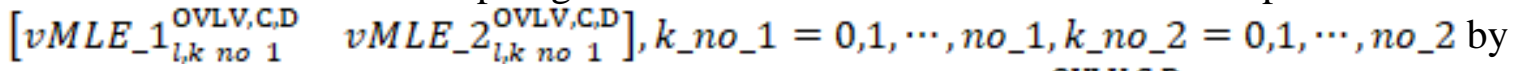
taking into consideration the horizontal spacings $\triangle M L E_{-} 1_{l}^{\text {OVLV,CD }}$ and vertical ones $\triangle M L E \_2_{l}^{\mathrm{OVLV} \mathrm{C}_{3} \mathrm{D}}$-see eqs. (4) and (5) of [43]. Fourth, step FL1.07 activates the random number generator of Phase D of Fig.2(a) that exploits the MLE pair combinations of the virtual indicative OV LV BPL topologies of virtual subclasses per CASD while $P$ members for each virtual OV LV BPL topology subclass are generated and added. Fifth, by activating the operation of Phases E and F of iSHM of Fig. 2(a), FL1.08 computes the

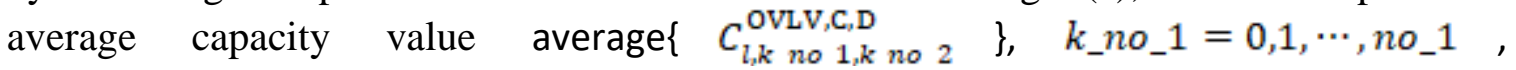
$k_{-}$no_2 $=0,1, \cdots$, no_2 of each virtual OV LV BPL topology subclass. By deploying Group 1.C, step FL1.09 receives as input all the $($ no_1 $1+1) \times($ no_2 +1$)$ MLE pair combinations of the virtual indicative OV LV BPL topologies of virtual subclasses per CASD

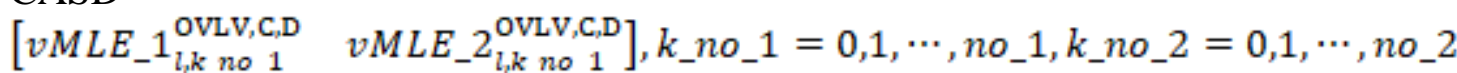


from the step FL1.06 of Group 1.B and provides the minimum and maximum values of horizontal and vertical axes of the 2D contour plot of the class map per CASD (i.e., CASD parameter mapping) while the step FL1.10 receives as input the CASD parameters from step FL1.09 and the average capacity values average $\left\{C_{l, k \text { no } 1_{2} k \text { no } 2}^{\text {OVLV }}\right\}$, $k_{-} n o \_1=0,1, \cdots, n o \_1, k_{-} n o_{-} 2=0,1, \cdots, n o \_2$ of all possible virtual OV LV BPL topology subclasses from step FL1.08 of Group 1.B and provides the existing CASD parameter map enriched with the average capacities of all possible virtual OV LV BPL topology subclasses. Finally, by synthesizing its inputs into a $2 \mathrm{D}$ contour plot, step FL1.11 receives the CASD parameter map from Group1.C and the capacities of

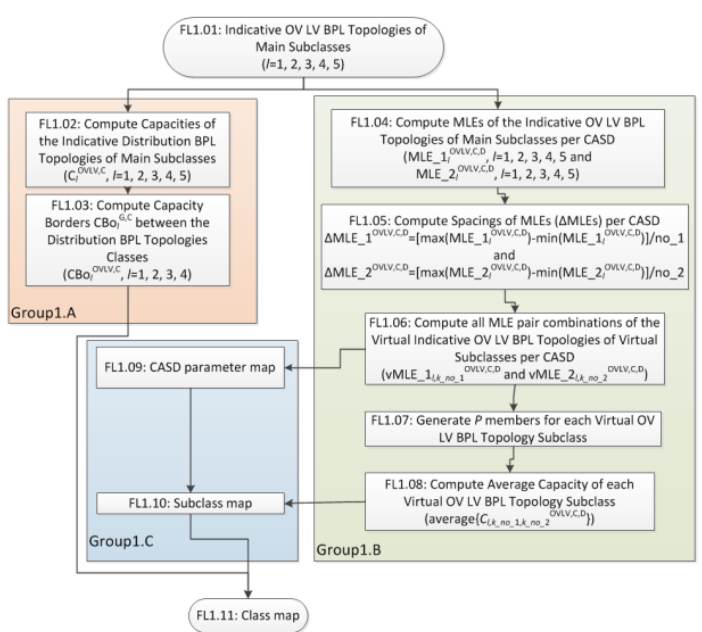

(a)

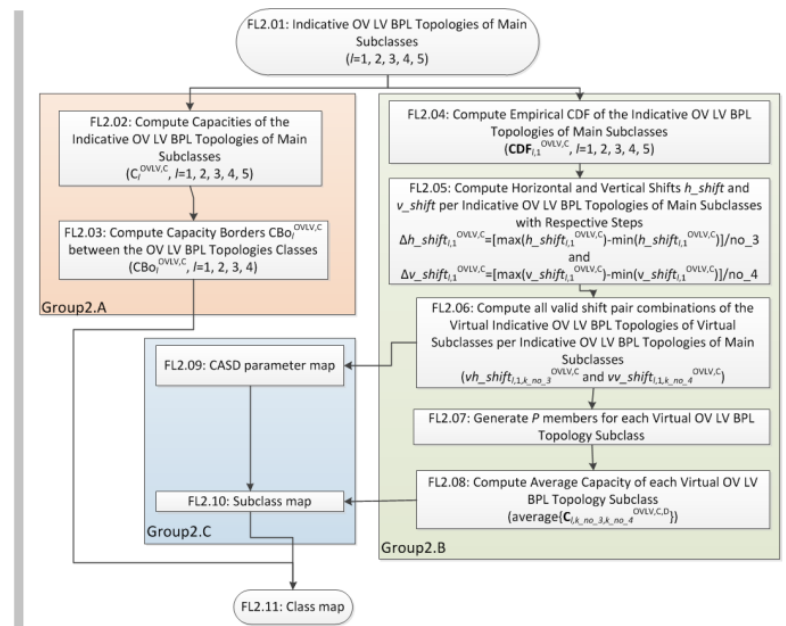

(b)

Fig. 3. Definition procedure flowcharts [43]. (a) iSHM. (b) mSHM.

the real indicative OV LV BPL topologies of main subclasses accompanied with the capacity borders between the adjacent OV LV BPL topology classes from Group1.A while step FL1.11 gives as output the class map that is the result of the definition procedure of iSHM.

Similarly to Sec.3.2, in accordance with [43] and with reference to Fig. 3(b), the operation of $\mathrm{mSHM}$ definition procedure can be described by simply highlighting the similarities and the differences between mSHM steps and groups and the respective ones of iSHM. Since the initialization of the definition procedure deals with the insertion of the real indicative OV LV BPL topologies of main subclasses of Table 1, step FL2.01 of $\mathrm{mSHM}$ and step FL1.01 remain the same. Also, Group 2.B of mSHM that consists of steps FL2.02 and FL2.03 deals with the capacities of the real indicative OV LV BPL topologies and the capacity borders between the adjacent OV LV BPL topology classes without any differences from the operation of Group 1.A of iSHM. The main differences between $\mathrm{mSHM}$ and iSHM definition procedures are concentrated in Group 2.B of mSHM and Group 1.B of iSHM; since mSHM adopts the Empirical CASD instead of the five CASDs of iSHM, step FL2.04 first computes the Empirical CDFs $\mathrm{CDF}_{l, 1}^{\text {OVLV,C }}, l=1,2,3,4,5$ of the real indicative OV LV BPL topologies of main 
subclasses. Then, step FL2.05 computes the repetition horizontal step $\Delta h_{-}$shift $t_{l, 1}^{\text {OVLV }, \mathrm{C}}, l=1,2,3,4,5$ and vertical step $\Delta v_{-}$shift $_{l, 1}^{\text {OVLV }, \mathrm{C}}, l=1,2,3,4,5$ with respect to eqs. (6) and (7) of [43] by taking into account the spacing of the horizontal shift $h_{-}$shift $t_{l_{1} 1}^{\text {OVLV } \mathrm{C} C}$ and vertical shift $v_{-}$shif $t_{l_{i} 1}^{\text {OVLV } \mathrm{CC}}$ of the Empirical CDFs of the real indicative OV LV BPL topologies of main subclasses where no_3 and no_4 are the number of spacings for the horizontal and vertical axis, respectively. Afterwards, step FL2.06 computes all $($ no_3 $3+1) \times($ no_4 +1$)$ shift pair combinations of the virtual indicative $\mathrm{OV} \quad \mathrm{LV}$ BPL topologies of virtual subclasses

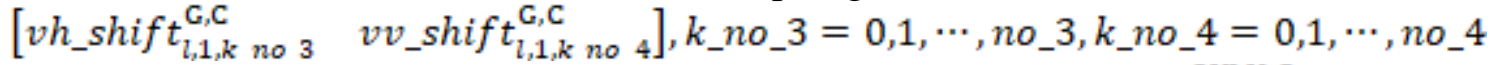
by taking into consideration the repetition horizontal step $\Delta h_{-}$shift $t_{l, 1}^{\text {OVLV,C }}$ and vertical one $\Delta v_{-}$shif $t_{l, 1}^{\text {OVLV }, \mathrm{C}}$-see eqs. (8) and (9) of [43]-. In fact, the aforementioned repetition steps and shifts are used so that the Empirical CDF of the virtual OV LV BPL topologies of subclasses can be computed with respect to the Empirical CDFs of the real indicative OV LV BPL topologies of main subclasses. Similarly to the steps of Group 1.B of iSHM definition procedure, step FL2.07 exploits the random number generator of Phase D of Fig. 2(b), step FL2.08 enriches each virtual OV LV BPL topology subclass with $P$ members and step FL2.09 computes the average capacity values average $\left\{C_{l, k \text { no } 3, k \text { no }{ }_{4}}^{\text {OVLV, }}\right\}$, $k_{-}$no_3 $=0,1, \cdots, n o \_3, k_{-}$no_ $4=0,1, \cdots$, no_4 of all possible virtual OV LV BPL topology subclasses of step FL2.08. As the Group 2.C and the step FL2.11 of mSHM definition procedure are concerned, these remain the same with the respective Group 1.C and step FL1.11 of iSHM definition procedure with the only difference that the axes of $\mathrm{mSHM}$ class maps that anyway the primary result of the described procedure comprise horizontal and vertical shifts while in the case of iSHM, the axes of iSHM class maps comprise CASD MLEs.

\subsection{Default Operation Settings for DHM, iSHM, mSHM, Definition Procedures and Class Maps}

Since DHM, iSHM, mSHM, definition procedures and class maps support an interconnected system, a set of default operation settings, which have already been reported in [35]-[37], are simply reported in this paper.

As the default operation settings for DHM are assumed, these are further divided into two categories; say, the topological and circuital operation setting categories. In the case of the default topological operation setting category, the real indicative OV LV BPL topologies that concern average long end-to-end connections of $1000 \mathrm{~m}$ have already been presented in Table 1. In the case of the default circuital operation settings, these have explicitly been detailed in [7], [8], [12], [15]-[17], [23], [25]-[27], [48], [52], [53], [55], [56], [63]-[72]. The default circuital operation settings comprise four assumptions regarding the interpretation of the indicative OV LV BPL topologies from DHM, namely: (i) the branching and distribution cables are assumed identical; (ii) the interconnections between the distribution and branch conductors are fully activated; (iii) the transmitting and the receiving ends are assumed to be matched to the characteristic impedance of the supported modal channels; and (iv) the branch terminations are treated as open circuit terminations. 
As the default operation settings for iSHM are assumed, these operation settings can be further divided into seven categories as already been outlined in [36], [44], namely: (i) BPL operation frequency range: The BPL operation frequency range that is considered in this paper is equal to $3-30 \mathrm{MHz}$ while 270 subchannels of $0.1 \mathrm{MHz}$ frequency spacing (i.e, $f_{s}=0.1 \mathrm{MHz}$ ) are considered so that the assumption of the flatfading subchannels, which is a typical scenario during the capacity computations in BPL channels, is valid [25]-[27], [30], [52], [56]; (ii) Default coupling scheme system: Already been mentioned in Sec.3.1, CS2 module, which has been detailed in [29], [59], can be considered as the default DHM coupling scheme module. Among the available coupling schemes that are supported by $\mathrm{CS} 2$ module, only $\mathrm{WtG}^{1}$ coupling scheme is assumed to be the default one so that a direct comparison between the results of this paper and those of [36] and [37], can be performed; (iii) Computation of the coupling scheme channel attenuation differences: To prevent the appearance of infinite terms during the application of Lognormal, Wald and Weibull CASDs in iSHM, the coupling scheme channel attenuation differences are assumed to be equal to an arbitrarily low value, say $1 \times 10^{-11}$, when zero or negative coupling scheme channel attenuation differences rarely occur; (iv) Members of each BPL topology subclass: 100 member OV LV BPL topologies (i.e., $P=100$ ) are going to be added in each BPL topology subclass per CASD in iSHM Phase D of Fig. 2(a); (v) IPSD limits: In accordance with Sec.3.1, FCC Part 15 is assumed to be the default EMI policy in this paper. In the frequency range $3-30 \mathrm{MHz}$ of interest, $-60 \mathrm{dBm} / \mathrm{Hz}$ are the FCC Part 15 IPSD limits $p($.$) suitable for the$ operation of OV LV BPL networks [25], [26], [73]; (vi) Noise PSD levels: Already been mentioned in Sec.3.1, FL noise model of [62], [74] is adopted for the capacity computations in the $3-30 \mathrm{MHz}$ frequency range [25], [26], [30], [52], [75]; say $-105 \mathrm{dBm} / \mathrm{Hz}$ is assumed to be the default AWGN PSD limit level $N(*)$ for OV LV BPL networks; and (vii) Best CASD with respect to its capacity estimation: This is one of the main objective of the companion paper of [76] to be determined for the OV LV BPL networks. Anyway, in accordance with [36], [37], it has been demonstrated for the iSHM that Weibull and Wald CASDs perform the best capacity estimations in OV MV and UN MV power grid types, respectively, regardless of the examined BPL topology subclass when the respective default operation settings concerning IPSD limits, noise PSD levels and applied coupling scheme are assumed. Seventh, in accordance with [36], [37], the performance of iSHM and the accuracy of its capacity results significantly depend on the selection of the CASD. Based on the findings of [36] and [45], it has been demonstrated for the iSHM that Weibull and Gaussian CASDs perform the best capacity estimations in OV MV and OV HV BPL networks, respectively, regardless of the examined BPL topology subclass when the aforementioned default operation settings are adopted. Anyway, one of the main interest of the companion paper of [76] is the identification of the best CASD for the OV LV BPL networks with respect to the best capacity estimations when the default operation settings are applied.

As the default operation settings for mSHM are assumed, these operation settings remain the same with the iSHM ones when the only difference lies in the seventh category (i.e., best CASD with respect to its capacity estimation). In contrast with iSHM, only one CASD, say, the Empirical CASD, is adopted by mSHM by default.

Finally, as the default operation settings of the definition procedures are concerned, these are further divided into two groups: (i) iSHM definition procedure 
default operation settings: With respect to FL1.05 of Fig. 3(a), the number of spacings for the horizontal and vertical axis (i.e., no_1 and no_2, respectively) is assumed to be equal to 10 in both cases. Note that the most suitable CASD with respect to its capacity estimation is going to be determined in [76] where the spacings for the horizontal and vertical axis are there applied; and (ii) $m S H M$ definition procedure default operation settings: Since Empirical CASD is the only examined CASD, CDFs are of interest and not MLEs. With respect to FL2.05 of Fig. 3(b), the number of spacings for the horizontal and vertical axis (i.e., no_3 and no_4, respectively) is assumed to be equal to 10 in both cases. Since CDF shifts are applied during the mSHM definition procedure, the maximum and minimum horizontal shift is assumed to be equal to $30 \mathrm{~dB}$ and $-30 \mathrm{~dB}$, respectively, while the maximum and minimum vertical shift is assumed to be equal to 1 and 0, respectively. Since the last step of both definition procedures (i.e., FL1.11 or FL2.11) deals with the class mapping, the aforementioned iSHM and mSHM definition procedure default operation settings have significant impact on the appearance of the class maps.

\section{TIM, FIIM and HS-DET method - The Basics and their Class Maps Footprints}

Class maps define the graphical basis where the footprints of the three smart grid broadband applications of interest (i.e., TIM, FIIM and HS-DET method) are going to be projected. In this Section, the three smart grid broadband applications are briefly outlined as well as the default operation settings that are required to be adopted. Note that numerical details concerning the default operation settings are also given in [76].

\subsection{TIM}

In accordance with [46], a BPL topology can be accurately identified with respect to its topological characteristics (i.e., number of branches, length of branches, length of main distribution lines and branch terminations) when TIM is applied. In fact, TIM can recognize the BPL topology even if significant measurement differences may occur by appropriately approximating the measured coupling scheme channel transfer function data of the BPL topology, which are contaminated by measurement differences. To approximate measured coupling scheme channel transfer function data, TIM exploits the application of L1PMA, which is a piecewise monotonic data approximation [77], [78], to its TIM BPL topology database where real BPL topologies with their respective theoretical and measured coupling scheme channel transfer functions stand. Depending on the required examined scenario, corresponding TIM BPL topology database specifications are concerned for the database preparation; say, the maximum number of branches $N$, the length spacing $L_{\mathrm{s}}$ for both branch distance and branch length and the maximum branch length $L_{b}$ for the OV LV BPL topologies.

In the companion paper, to illustrate the TIM footprint on the class maps of all possible real OV LV BPL topologies, the OV LV BPL topology database can be appropriately adjusted when the measurement differences are assumed to be equal to zero. For each real OV LV BPL topology of the TIM BPL topology database and with reference to its coupling scheme channel transfer function data, the following 
corresponding records are inserted: (i) the MLEs of the supported CASDs as described in iSHM; (ii) the Empirical CDF as described in mSHM; and (iii) the capacity; when the default operation settings of Sec.3.4 are assumed.

With reference to Sec.3.3, each OV LV BPL topology of the TIM BPL topology database can be represented on the iSHM class map for given CASD by appropriately exploiting its respective CASD MLEs. Therefore, the TIM footprint on the iSHM class map consists of all TIM database OV LV BPL topology points, which are expected to create a subarea on the entire class map since class maps consist of all possible OV LV BPL topologies (i.e., virtual OV LV BPL topologies) in terms of the corresponding CASD MLEs. As the capacity of each OV LV BPL topology of the TIM BPL topology database is concerned, its capacity remains very close to the average capacity of the OV LV BPL topology subclass whose virtual indicative OV LV BPL topology is characterized by the same CASD MLEs with the ones of real OV LV BPL topology of the TIM database when the examined CASD performs successful capacity estimation in terms of the percentage change metric [36], [37].

With reference to Sec.3.3, each OV LV BPL topology of the TIM BPL topology database cannot be directly represented on the mSHM class map for given real indicative OV LV BPL topology of the main subclasses but a capacity correlation should be first computed so that the best fit between the examined OV LV BPL topology of the TIM BPL topology database and one horizontally and vertically shifted version of the real indicative OV LV BPL topology (i.e., virtual indicative OV LV BPL topologies) may occur. Strictly theoretically, with reference to eq. (3), the aforementioned best fit is achieved through the minimization of the following Frobenius distance with regards to the subchannel capacities, namely:

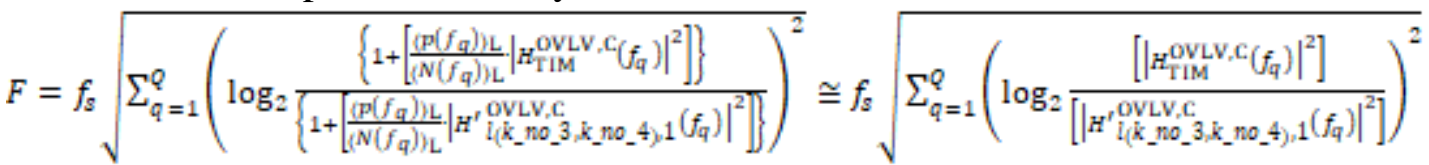

where $H_{\mathrm{TIM}}^{\mathrm{OVL} V_{S} \mathrm{C}}\left(f_{q}\right)$ is the $q$-th element of the $1 \times Q$ coupling scheme channel transfer

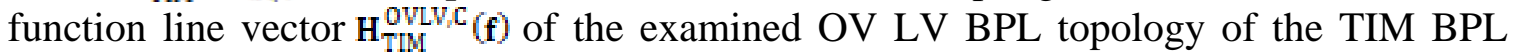

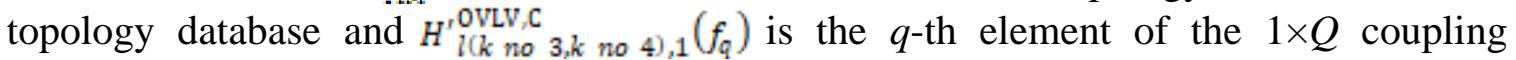

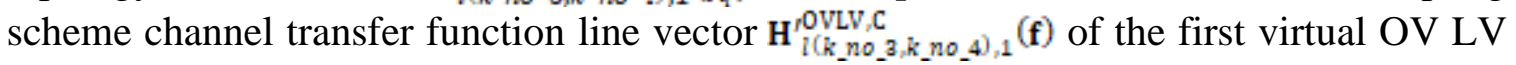
BPL topology of mSHM that corresponds to the [ $k_{-}$no_3 $k_{-}$no_4] shift pair combination. The minimization of the Frobenius distance of eq.(7) implies that the capacity of the examined OV LV BPL topology of the TIM BPL topology database remains almost equal to the capacity of the shifted version of the real indicative OV LV BPL topology with [ $\left.k_{-} n o_{-} 3 \quad k_{-} n o \_4\right]$ shift pair combination (say, best shifted version of the real indicative OV LV BPL topology). Practically, the best shifted version of the real indicative OV LV BPL topology can approximately be computed by comparing the capacity of the examined OV LV BPL topology of the TIM BPL topology database with all the capacities of the virtual OV LV BPL topologies and finding the corresponding [ $k_{-} n o_{-} 3 k_{-}$no_4] shift pair combination (i.e., practical approximation of Frobenius distance). Thus, the examined OV LV BPL topology of the TIM BPL topology database can be indirectly characterized by the $\left[\begin{array}{ll}k_{-} n o_{-} 3 & k_{-} n o \_4\end{array}\right]$ shift pair combination of the best shifted version of the real indicative OV LV BPL topology and, hence, can be represented on the mSHM class map at the respective coordinates of the horizontal and vertical shifts. Similarly to the TIM footprint on the iSHM class map, the TIM footprint 
on the mSHM class map consists of all TIM database OV LV BPL topology points, creating a subarea on the entire class map. Similarly to iSHM class maps, the capacity of the OV LV BPL topologies of the TIM BPL topology database remain very close to the average capacity of the OV LV BPL topology subclass whose virtual indicative OV LV BPL topology is characterized by an Empirical CDF that almost coincide with the one of real OV LV BPL topology of the TIM database when the Empirical CASD performs successful capacity estimation in terms of the percentage change metric [36], [37].

\subsection{FIIM}

In accordance with [46], FIIM achieves to identify any faults and instabilities that may occur in BPL topologies. FIIM repertory of faults and instabilities can be divided into two categories with two subcategories each, namely [79]: (i) Faults: This category describes all the interruptions that may occur across the lines of the power grid and can be further divided into two subcategories of line interruptions, say Fault in transmission line and Fault in branch line; and (ii) Instabilities: This category describes all the failures that can occur in the equipment across the power grid and can be further divided into two subcategories, say Instability in branch interconnections and Instability in branch terminations. Similarly to TIM, FIIM applies L1PMA to the measured coupling scheme transfer function data while FIIM supports its own FIIM BPL topology database where real BPL topologies that suffer from all the aforementioned faults and instabilities with their respective theoretical and measured coupling scheme channel transfer functions stand. In this paper, only the subcategory of one sole fault in one branch line is going to be examined. For given indicative OV LV BPL topology of Table 1, as the FIIM OV LV BPL topology database specifications are concerned for the database preparation, these remain the same with the TIM OV LV BPL topology database as described in Sec.4.1 while the fault location at the existing branch of the indicative OV LV BPL topology ranges from $0.1 \mathrm{~m}$ to the end of the corresponding branch with a step of $0.1 \mathrm{~m}$. Note that the faulty branch termination is assumed to be open-circuit.

Similarly to the examined TIM iSHM and mSHM footprints of Sec.4.1, OV LV BPL topologies of the FIIM BPL topology database with one sole fault in one branch line can be represented on the iSHM class map for given CASD and indicative OV LV BPL topology of Table 1 by appropriately exploiting their respective CASD MLEs (FIIM iSHM footprint). As the FIIM mSHM footprint is concerned, by applying the practical approximation of Frobenius distance, virtual OV LV BPL topologies, which are characterized by respective [ $\left.k_{-} n o_{-} 3 \quad k_{-} n o_{-} 4\right]$ shift pair combinations, that approximate the behavior of the corresponding examined OV LV BPL topologies of the FIIM BPL topology database with one sole fault in one branch line can be identified (corresponding best fits). Hence, OV LV BPL topologies of the FIIM BPL topology database with one sole fault in one branch line can be represented on the mSHM class map at the coordinates of the horizontal and vertical shifts of their corresponding best fits (FIIM $\mathrm{mSHM}$ footprint).

\subsection{HS-DET Method}

In accordance with [47], HS-DET method achieves to identify any hook style energy theft in OV LV BPL networks either in theoretical or in real operation conditions; say with or without measurement differences, respectively. In [80], [81], the detection efficiency of HS-DET method has been exhaustively verified even in special operation cases of OV LV 
BPL networks (e.g., high measurement differences, very long hooks, "smart" hooks, feint "smart" hooks and hook interconnection issues). In contrast with TIM and FIIM, HS-DET method does not apply piecewise monotonic data approximations but adopts the metric of PES between the results collected by in-situ measurements and the theoretical results derived from DHM that occur during the normal operation of channel attenuation determination -see eqs.(3)-(8) of [47]-. Since the measurement differences are neglected during the determination of the coupling scheme channel transfer functions in this paper, PES is computed between the theoretical coupling scheme transfer functions of the real OV LV BPL topology of the TIM BPL topology database and the corresponding OV LV BPL topology that suffers from the hook style energy theft. Note that one sole hook is assumed during the energy theft from the real OV LV BPL topology but a plethora of different variations of the real OV LV BPL topology can be examined in terms of the hook distance from the transmitting end and the hook length. Hence, for given real OV LV BPL topology of the TIM BPL topology database, HS-DET method BPL topology database consists of all the possible corresponding real OV LV BPL topology with hook that can be assumed when the length spacing for both hook distance and hook length is assumed as well as the maximum hook length $L_{b}$. In accordance with [47], except for the aforementioned assumptions concerning the HS-DET method BPL topology database specifications, the hook termination is assumed to be open circuit while the hook interconnection with the distribution lines is assumed to be complete (i.e., hook derivation points at the same distance from the transmitting end on all the three phases) and horizontal. Therefore, the hook can be treated by DHM as a branch. As already been verified in [80], [81], the previous assumptions are made in order to simplify the following analysis without losing its generality.

Similarly to the examined FIIM iSHM and mSHM footprints of Sec.4.2, OV LV BPL topologies of the HS-DET method BPL topology database with one sole hook can be represented on the iSHM class map for given CASD and real indicative OV LV BPL topology of Table 1, by appropriately exploiting their respective CASD MLEs (HS-DET method iSHM footprint). As the HS-DET method mSHM footprint is concerned, by applying the practical approximation of Frobenius distance, virtual OV LV BPL topologies, which are characterized by respective [ $k_{-}$no_3 $k_{-}$no_4$]$shift pair combinations, that approximate the behavior of the corresponding examined OV LV BPL topologies of the HS-DET method BPL topology database with one sole hook can be identified (corresponding best fits). Hence, OV LV BPL topologies of the HS-DET method BPL topology database with one sole hook can be represented on the mSHM class map at the coordinates of the horizontal and vertical shifts of their corresponding best fits (HS-DET method mSHM footprint).

\section{Conclusions}

In this paper, a review concerning the interoperability of DHM, iSHM, mSHM, the definition procedure and the class maps has been first presented for OV LV BPL networks as well as the required default settings for their fine combined operation. However, the main interest of this paper has focused on the theory presentation of the $\mathrm{OV}$ LV BPL topology footprints of TIM, FIIM and HS-DET method on the class maps rather than on the numerical evaluation that is demonstrated in [76]. With respect to specific settings concerning the applied OV LV BPL topology databases of TIM, FIIM and HS- 
DET method, the following footprint cases have been theoretically defined, namely: (i) iSHM and mSHM footprints of the real OV LV BPL topologies as described in TIM database; (ii) iSHM and mSHM footprints of OV LV BPL topologies of the FIIM BPL topology database with one sole fault in one branch line for given real indicative OV LV BPL topology; and (iii) iSHM and mSHM footprints of OV LV BPL topologies of the HS-DET method BPL topology database with one sole hook for given real indicative OV LV BPL topology. In [76], the numerical results concerning the first application of the combined operation of iSHM, mSHM, the definition procedure and the class maps on OV LV BPL topologies are demonstrated as well as the proposal of the aforementioned footprint cases.

\section{CONFLICTS OF INTEREST}

The author declares that there is no conflict of interests regarding the publication of this paper.

\section{References}

[1] M. H. Rehmani, M. Reisslein, A. Rachedi, M. Erol-Kantarci, and M. Radenkovic, "Integrating renewable energy resources into the smart grid: recent developments in information and communication technologies," IEEE Transactions on Industrial Informatics, vol. 14, no. 7, pp. 2814-2825, 2018.

[2] F. R. Yu, P. Zhang, W. Xiao, and P. Choudhury, "Communication systems for grid integration of renewable energy resources," IEEE Network, vol. 25, no. 5, pp. 22-29, Sep. 2011.

[3] B. Heile, "Smart grids for green communications [industry perspectives]," IEEE Wireless Commun., vol. 17, no. 3, pp. 4-6, Jun. 2010.

[4] G. Artale, A. Cataliotti, V. Cosentino, D. Di Cara, R. Fiorelli, S. Guaiana, and G. Tinè, "A New Low Cost Coupling System for Power Line Communication on Medium Voltage Smart Grids," IEEE Trans. on Smart Grid, vol. 9, no. 4, pp. 3321-3329, 2018.

[5] L. González-Sotres, C. Mateo, P. Frías, C. Rodríguez-Morcillo, and J. Matanza, "Replicability Analysis of PLC PRIME Networks for Smart Metering Applications," IEEE Trans. on Smart Grid, vol. 9, no. 2, pp. 827-835, Mar. 2018.

[6] A. ElSamadouny, A. El Shafie, M. Abdallah, and N. Al-Dhahir, "Secure SumRate-Optimal MIMO Multicasting Over Medium-Voltage NB-PLC Networks," IEEE Trans. on Smart Grid, vol. 9, no. 4, pp. 2954-2963, Jul. 2018.

[7] A. G. Lazaropoulos and P. G. Cottis, "Transmission characteristics of overhead medium voltage power line communication channels," IEEE Trans. Power Del., vol. 24, no. 3, pp. 1164-1173, Jul. 2009.

[8] A. G. Lazaropoulos and P. G. Cottis, "Broadband transmission via underground medium-voltage power lines-Part I: transmission characteristics," IEEE Trans. Power Del., vol. 25, no. 4, pp. 2414-2424, Oct. 2010. 
[9] E. Biglieri, "Coding and modulation for a horrible channel," IEEE Commun. Mag., vol. 41, no. 5, pp. 92-98, May 2003.

[10] M. Gebhardt, F. Weinmann, and K. Dostert, "Physical and regulatory constraints for communication over the power supply grid," IEEE Commun. Mag., vol. 41, no. 5, pp. 84-90, May 2003.

[11] M. Götz, M. Rapp, and K. Dostert, "Power line channel characteristics and their effect on communication system design," IEEE Commun. Mag., vol. 42, no. 4, pp. 78-86, Apr. 2004.

[12] A. G. Lazaropoulos, "Towards Modal Integration of Overhead and Underground Low-Voltage and Medium-Voltage Power Line Communication Channels in the Smart Grid Landscape: Model Expansion, Broadband Signal Transmission Characteristics, and Statistical Performance Metrics (Invited Paper)," ISRN Signal Processing, vol. 2012, Article ID 121628, pp. 1-17, 2012. [Online]. Available: http://www.hindawi.com/isrn/sp/2012/121628/

[13] K. Dostert, Powerline Communications. Upper Saddle River, NJ: Prentice-Hall, 2001.

[14] F. Versolatto and A. M. Tonello, "An MTL theory approach for the simulation of MIMO power-line communication channels," IEEE Trans. Power Del., vol. 26, no. 3, pp. 1710-1717, Jul. 2011.

[15] P. Amirshahi and M. Kavehrad, "High-frequency characteristics of overhead multiconductor power lines for broadband communications," IEEE J. Sel. Areas Commun., vol. 24, no. 7, pp. 1292-1303, Jul. 2006.

[16] T. Sartenaer, "Multiuser communications over frequency selective wired channels and applications to the powerline access network" Ph.D. dissertation, Univ. Catholique Louvain, Louvain-la-Neuve, Belgium, Sep. 2004.

[17] T. Sartenaer and P. Delogne, "Deterministic modelling of the (Shielded) outdoor powerline channel based on the multiconductor transmission line equations," IEEE J. Sel. Areas Commun., vol. 24, no. 7, pp. 1277-1291, Jul. 2006.

[18] T. Calliacoudas and F. Issa, "“Multiconductor transmission lines and cables solver," An efficient simulation tool for plc channel networks development," presented at the IEEE Int. Conf. Power Line Communications and Its Applications, Athens, Greece, Mar. 2002.

[19] S. Galli and T. Banwell, "A deterministic frequency-domain model for the indoor power line transfer function," IEEE J. Sel. Areas Commun., vol. 24, no. 7, pp. 1304-1316, Jul. 2006.

[20] S. Galli and T. Banwell, "A novel approach to accurate modeling of the indoor power line channel-Part II: Transfer function and channel properties," IEEE Trans. Power Del., vol. 20, no. 3, pp. 1869-1878, Jul. 2005.

[21] A. Pérez, A. M. Sánchez, J. R. Regué, M. Ribó, R. Aquilué, P. RodréguezCepeda, and F. J. Pajares, "Circuital and modal characterization of the power-line network in the PLC band," IEEE Trans. Power Del., vol. 24, no. 3, pp. 11821189, Jul. 2009.

[22] H. Meng, S. Chen, Y. L. Guan, C. L. Law, P. L. So, E. Gunawan, and T. T. Lie, "Modeling of transfer characteristics for the broadband power line communication channel,”IEEE Trans. Power Del., vol. 19, no. 3, pp. 1057-1064, Jul. 2004. 
[23] P. Amirshahi, "Broadband access and home networking through powerline networks" Ph.D. dissertation, Pennsylvania State Univ., University Park, PA, May 2006.

[24] A. G. Lazaropoulos, “Towards Broadband over Power Lines Systems Integration: Transmission Characteristics of Underground Low-Voltage Distribution Power Lines," Progress in Electromagnetics Research B, vol. 39, pp. 89-114, 2012. [Online]. Available: http://www.jpier.org/PIERB/pierb39/05.12012409.pdf

[25] A. G. Lazaropoulos and P. G. Cottis, "Capacity of overhead medium voltage power line communication channels," IEEE Trans. Power Del., vol. 25, no. 2, pp. 723-733, Apr. 2010.

[26] A. G. Lazaropoulos and P. G. Cottis, "Broadband transmission via underground medium-voltage power lines-Part II: capacity," IEEE Trans. Power Del., vol. 25, no. 4, pp. 2425-2434, Oct. 2010.

[27] A. G. Lazaropoulos, "Broadband transmission and statistical performance properties of overhead high-voltage transmission networks," Hindawi Journal of Computer Networks and Commun., 2012, article ID 875632, 2012. [Online]. Available: http://www.hindawi.com/journals/jcnc/aip/875632/

[28] A. G. Lazaropoulos, "Wireless Sensor Network Design for Transmission Line Monitoring, Metering and Controlling Introducing Broadband over PowerLinesenhanced Network Model (BPLeNM)," ISRN Power Engineering, vol. 2014, Article ID 894628, 22 pages, 2014. doi:10.1155/2014/894628. [Online]. Available: http://www.hindawi.com/journals/isrn.power.engineering/2014/894628/

[29] A. G. Lazaropoulos, "Broadband Performance Metrics and Regression Approximations of the New Coupling Schemes for Distribution Broadband over Power Lines (BPL) Networks," Trends in Renewable Energy, vol. 4, no. 1, pp. 43-73, Jan. $2018 . \quad$ [Online]. Available: http://futureenergysp.com/index.php/tre/article/view/59/pdf

[30] A. G. Lazaropoulos, "Smart Energy and Spectral Efficiency (SE) of Distribution Broadband over Power Lines (BPL) Networks - Part 1: The Impact of Measurement Differences on SE Metrics," Trends in Renewable Energy, vol. 4, no. 2, pp. 125-184, Aug. 2018. [Online]. Available: http://futureenergysp.com/index.php/tre/article/view/76/pdf

[31] A. M. Tonello and T. Zheng, "Bottom-up transfer function generator for broadband PLC statistical channel modeling," In Proc. IEEE International Symposium on Power Line Communications and Its Applications 2009, ISPLC 2009, pp. 7-12, Mar. 2009.

[32] A. M. Tonello and F. Versolatto, "Bottom-up statistical PLC channel modelingPart I: Random topology model and efficient transfer function computation," IEEE Transactions on Power Delivery, vol. 26, no. 2, pp. 891-898, 2011.

[33] A. Pittolo and A. M. Tonello, "A synthetic statistical mimo plc channel model applied to an in-home scenario," IEEE Transactions on Communications, vol. 65, no. 6, pp. 2543-2553, 2017.

[34] A. M. Tonello and F. Versolatto, "Bottom-up statistical PLC channel modelingPart II: Inferring the statistics," IEEE transactions on Power Delivery, vol. 25, no. 4, pp. 2356-2363, 2010. 
[35] A. G. Lazaropoulos, "Statistical Broadband over Power Lines Channel Modeling - Part 1: The Theory of the Statistical Hybrid Model," Progress in Electromagnetics Research C, vol. 92, pp. 1-16, 2019. [Online]. Available: http://www.jpier.org/PIERC/pierc92/01.19012902.pdf

[36] A. G. Lazaropoulos, "Statistical Broadband over Power Lines (BPL) Channel Modeling - Part 2: The Numerical Results of the Statistical Hybrid Model," Progress in Electromagnetics Research C, vol. 92, pp. 17-30, 2019. [Online]. Available: http://www.jpier.org/PIERC/pierc92/02.19012903.pdf

[37] A. G. Lazaropoulos, "Enhancing the Statistical Hybrid Model Performance in Overhead and Underground Medium Voltage Broadband over Power Lines Channels by Adopting Empirical Channel Attenuation Statistical Distribution," Trends in Renewable Energy, vol. 5, no. 2, pp. 181-217, 2019. [Online]. Available: http://futureenergysp.com/index.php/tre/article/view/96/pdf

[38] T. Oliveira, A. Picorone, C. Zeller, S. Netto, and M. Ribeiro, "Statistical Modeling of Brazilian In-Home PLC Channel Features," Journal of Communication and Information Systems, vol. 34, no. 1, pp. 154-168, 2019.

[39] T. R. Oliveira, C. B. Zeller, S. L. Netto, and M. V. Ribeiro, "Statistical modeling of the average channel gain and delay spread in in-home PLC channels," in Proc. in IEEE International Symposium on Power Line Communications and Its Applications, pp. 184-188, Mar. 2015.

[40] J. A. Cortes, F. J. Canete, L. Diez, and J. L. G. Moreno, "On the statistical properties of indoor power line channels: Measurements and models," in Proc. IEEE International Symposium on Power Line Communications and Its Applications, pp. 271-276, Apr. 2011.

[41] A. M. Tonello, F. Versolatto, and A. Pittolo, "In-home power line communication channel: Statistical characterization," IEEE Trans. on Commun., vol. 62, no. 6, pp. 2096-2106, Jun. 2014.

[42] S. Galli, "A novel approach to the statistical modeling of wireline channels," IEEE Transactions on Commun., vol. 59, no. 5, pp. 1332-1345, May 2011.

[43] A. G. Lazaropoulos, "Virtual Indicative Broadband over Power Lines Topologies for Respective Subclasses by Adjusting Channel Attenuation Statistical Distribution Parameters of Statistical Hybrid Models - Part 1: Theory," Trends in Renewable Energy, vol. 5, no. 3, pp. 237-257, Aug. 2019. [Online]. Available: http://futureenergysp.com/index.php/tre/article/view/99/pdf

[44] A. G. Lazaropoulos, "Virtual Indicative Broadband over Power Lines Topologies for Respective Subclasses by Adjusting Channel Attenuation Statistical Distribution Parameters of Statistical Hybrid Models - Part 2: Numerical Results for the Overhead and Underground Medium-Voltage Power Grids," Trends in Renewable Energy, vol. 5, no. 3, pp. 258-281, Aug. 2019. [Online]. Available: http://futureenergysp.com/index.php/tre/article/view/100/pdf

[45] A. G. Lazaropoulos, "Virtual Indicative Broadband over Power Lines Topologies for Respective Subclasses by Adjusting Channel Attenuation Statistical Distribution Parameters of Statistical Hybrid Models - Part 3: The Case of Overhead Transmission Power Grids," Trends in Renewable Energy, vol. 5, no. 3, pp. 282-306, Aug. 2019. [Online]. Available: http://futureenergysp.com/index.php/tre/article/view/101/pdf 
[46] A. G. Lazaropoulos, "Improvement of Power Systems Stability by Applying Topology Identification Methodology (TIM) and Fault and Instability Identification Methodology (FIIM)-Study of the Overhead Medium-Voltage Broadband over Power Lines (OV MV BPL) Networks Case," Trends in Renewable Energy, vol. 3, no. 2, pp. 102-128, Apr. 2017. [Online]. Available: http://futureenergysp.com/index.php/tre/article/view/34

[47] A. G. Lazaropoulos, "Detection of Energy Theft in Overhead Low-Voltage Power Grids - The Hook Style Energy Theft in the Smart Grid Era," Trends in Renewable Energy, vol. 5, no. 1, pp. 12-46, Oct. 2018. [Online]. Available: http://futureenergysp.com/index.php/tre/article/view/81/pdf

[48] OPERA1, D44: Report presenting the architecture of plc system, the electricity network topologies, the operating modes and the equipment over which PLC access system will be installed, IST Integr. Project No 507667, Dec. 2005.

[49] M. D'Amore and M. S. Sarto, "A new formulation of lossy ground return parameters for transient analysis of multiconductor dissipative lines," IEEE Trans. Power Del., vol. 12, no. 1, pp. 303-314, Jan. 1997.

[50] M. D'Amore and M. S. Sarto, "Simulation models of a dissipative transmission line above a lossy ground for a wide-frequency range-Part I: Single conductor configuration," IEEE Trans. Electromagn. Compat., vol. 38, no. 2, pp. 127-138, May 1996.

[51] M. D'Amore and M. S. Sarto, "Simulation models of a dissipative transmission line above a lossy ground for a wide-frequency range-Part II: Multi-conductor configuration," IEEE Trans. Electromagn. Compat., vol. 38, no. 2, pp. 139-149, May 1996.

[52] A. G. Lazaropoulos, "Review and Progress towards the Capacity Boost of Overhead and Underground Medium-Voltage and Low-Voltage Broadband over Power Lines Networks: Cooperative Communications through Two- and ThreeHop Repeater Systems," ISRN Electronics, vol. 2013, Article ID 472190, pp. 1$\begin{array}{lll}19, & 2013 . & \text { [Online]. }\end{array}$ http://www.hindawi.com/isrn/electronics/aip/472190/

[53] A. G. Lazaropoulos, "Broadband over Power Lines (BPL) Systems Convergence: Multiple-Input Multiple-Output (MIMO) Communications Analysis of Overhead and Underground Low-Voltage and Medium-Voltage BPL Networks (Invited Paper)," ISRN Power Engineering, vol. 2013, Article ID 517940, pp. 1-30, 2013. [Online].

Available: http://www.hindawi.com/isrn/power.engineering/2013/517940/

[54] A. G. Lazaropoulos, "Main Line Fault Localization Methodology (MLFLM) in Smart Grid-The Underground Medium- and Low-Voltage Broadband over Power Lines Networks Case," Trends in Renewable Energy, vol. 4, no. 1, pp. 15-42, Dec. $2017 . \quad$ [Online]. Available: http://futureenergysp.com/index.php/tre/article/view/45

[55] A. G. Lazaropoulos, "Underground Distribution BPL Connections with $(\mathrm{N}+1)-$ hop Repeater Systems: A Novel Capacity Mitigation Technique," Elsevier Computers and Electrical Engineering, vol. 40, pp. 1813-1826, 2014.

[56] A. G. Lazaropoulos, "Deployment Concepts for Overhead High Voltage Broadband over Power Lines Connections with Two-Hop Repeater System: 
Capacity Countermeasures against Aggravated Topologies and High Noise Environments," Progress in Electromagnetics Research B, vol. 44, pp. 283-307, 2012. [Online]. Available: http://www.jpier.org/PIERB/pierb44/13.12081104.pdf

[57] C. R. Paul, Analysis of Multiconductor Transmission Lines. New York: Wiley, 1994.

[58] J. A. B. Faria, Multiconductor Transmission-Line Structures: Modal Analysis Techniques. New York: Wiley, 1994.

[59] A. G. Lazaropoulos, "New Coupling Schemes for Distribution Broadband over Power Lines (BPL) Networks," Progress in Electromagnetics Research B, vol. 71, pp. 39-54, 2016. [Online]. Available: http://www.jpier.org/PIERB/pierb71/02.16081503.pdf

[60] NTIA, "Potential interference from broadband over power line (BPL) systems to federal government radio communications at 1.7-80 MHz Phase 1 Study Vol. 1," NTIA Rep. 04-413, Apr. 2004.

[61] NATO, "HF Interference, Procedures and Tools (Interférences HF, procédures et outils) Final Report of NATO RTO Information Systems Technology," RTO-TRISTR-050, Jun. 2007.

[62] A. G. Lazaropoulos, "The Impact of Noise Models on Capacity Performance of Distribution Broadband over Power Lines Networks," Hindawi Computer Networks and Communications, vol. 2016, Article ID 5680850, 14 pages, 2016. doi:10.1155/2016/5680850. [Online].
http://www.hindawi.com/journals/jenc/2016/5680850/

[63] N. Suljanović, A. Mujčić, M. Zajc, and J. F. Tasič, "Approximate computation of high-frequency characteristics for power line with horizontal disposition and middle-phase to ground coupling," Elsevier Electr. Power Syst. Res., vol. 69, pp. 17-24, Jan. 2004.

[64] OPERA1, D5: Pathloss as a function of frequency, distance and network topology for various LV and MV European powerline networks. IST Integrated Project No 507667, Apr. 2005.

[65] N. Suljanović, A. Mujčić, M. Zajc, and J. F. Tasič, "High-frequency characteristics of high-voltage power line," in Proc. IEEE Int. Conf. on Computer as a Tool, Ljubljana, Slovenia, Sep. 2003, pp. 310-314.

[66] N. Suljanović, A. Mujčić, M. Zajc, and J. F. Tasič, "Power-line high-frequency characteristics: analytical formulation," in Proc. Joint 1st Workshop on Mobile Future \& Symposium on Trends in Communications, Bratislava, Slovakia, Oct. 2003, pp. 106-109.

[67] W. Villiers, J. H. Cloete, and R. Herman, "The feasibility of ampacity control on HV transmission lines using the PLC system," in Proc. IEEE Conf. Africon, George, South Africa, Oct. 2002, vol. 2, pp. 865-870.

[68] J. Anatory, N. Theethayi, R. Thottappillil, M. M. Kissaka, and N. H. Mvungi, "The influence of load impedance, line length, and branches on underground cable Power-Line Communications (PLC) systems," IEEE Trans. Power Del., vol. 23, no. 1, pp. 180-187, Jan. 2008.

[69] J. Anatory, N. Theethayi, and R. Thottappillil, "Power-line communication channel model for interconnected networks-Part II: Multiconductor system," IEEE Trans. Power Del., vol. 24, no. 1, pp. 124-128, Jan. 2009. 
[70] J. Anatory, N. Theethayi, R. Thottappillil, M. M. Kissaka, and N. H. Mvungi, "The effects of load impedance, line length, and branches in typical low-voltage channels of the BPLC systems of developing countries: transmission-line analyses," IEEE Trans. Power Del., vol. 24, no. 2, pp. 621-629, Apr. 2009.

[71] T. Banwell and S. Galli, "A novel approach to accurate modeling of the indoor power line channel-Part I: Circuit analysis and companion model," IEEE Trans. Power Del., vol. 20, no. 2, pp. 655-663, Apr. 2005.

[72] W. Villiers, J. H. Cloete, L. M. Wedepohl, and A. Burger, "Real-time sag monitoring system for high-voltage overhead transmission lines based on powerline carrier signal behavior," IEEE Trans. Power Del., vol. 23, no. 1, pp. 389-395, Jan. 2008.

[73] A. G. Lazaropoulos, "A Panacea to Inherent BPL Technology Deficiencies by Deploying Broadband over Power Lines (BPL) Connections with Multi-Hop Repeater Systems," Bentham Recent Advances in Electrical \& Electronic Engineering, vol. 10, no. 1, pp. 30-46, 2017

[74] A. G. Lazaropoulos, "Capacity Performance of Overhead Transmission MultipleInput Multiple-Output Broadband over Power Lines Networks: The Insidious Effect of Noise and the Role of Noise Models (Invited Paper)," Trends in Renewable Energy, vol. 2, no. 2, pp. 61-82, Jun. 2016. [Online]. Available: http://futureenergysp.com/index.php/tre/article/view/23

[75] A. G. Lazaropoulos, "Smart Energy and Spectral Efficiency (SE) of Distribution Broadband over Power Lines (BPL) Networks - Part 2: L1PMA, L2WPMA and L2CXCV for SE against Measurement Differences in Overhead Medium-Voltage BPL Networks," Trends in Renewable Energy, vol. 4, no. 2, pp. 185-212, Aug. $2018 . \quad$ [Online].

Available: http://futureenergysp.com/index.php/tre/article/view/77/pdf

[76] A. G. Lazaropoulos, "Statistical Channel Modeling of Overhead Low Voltage Broadband over Power Lines (OV LV BPL) Networks - Part 2: The Numerical Results of Class Map Footprints of Real OV LV BPL Topologies, Branch Line Faults and Hook Style Energy Thefts," Trends in Renewable Energy, vol. 6, no. 1, pp. 88109.

[77] I. C. Demetriou, "An application of best $L 1$ piecewise monotonic data approximation to signal restoration," IAENG International Journal of Applied Mathematics, vol. 53, no. 4, pp. 226-232, 2013.

[78] I. C. Demetriou, "L1PMA: A Fortran 77 Package for Best L1 Piecewise Monotonic Data Smoothing," Computer Physics Communications, vol. 151, no. 1, pp. 315-338, 2003.

[79] A. G. Lazaropoulos, "Measurement Differences, Faults and Instabilities in Intelligent Energy Systems - Part 2: Fault and Instability Prediction in Overhead High-Voltage Broadband over Power Lines Networks by Applying Fault and Instability Identification Methodology (FIIM)," Trends in Renewable Energy, vol. 2, no. 3, pp. 113-142, Oct. 2016. [Online]. Available: http://futureenergysp.com/index.php/tre/article/view/27/33

[80] A. G. Lazaropoulos, "Special Cases during the Detection of the Hook Style Energy Theft in Overhead Low-Voltage Power Grids through HS-DET Method - Part 1: High Measurement Differences, Very Long Hook Technique and "Smart" Hooks," 
Trends in Renewable Energy, vol. 5, no. 1, pp. 60-89, Jan. 2019. [Online]. Available: http://futureenergysp.com/index.php/tre/article/view/82/pdf

[81] A. G. Lazaropoulos, "Special Cases during the Detection of the Hook Style Energy Theft in Overhead Low-Voltage Power Grids through HS-DET Method - Part 2: Different Measurement Differences, Feint "Smart" Hooks and Hook Interconnection Issues," Trends in Renewable Energy, vol. 5, no. 1, pp. 90-116, Jan. 2019. [Online]. Available: http://futureenergysp.com/index.php/tre/article/view/83/pdf

Article copyright: (C) 2020 Athanasios G. Lazaropoulos. This is an open access article distributed under the terms of the Creative Commons Attribution 4.0 International License, which permits unrestricted use and distribution provided the original author and source are credited. 